\title{
Les processus de mise en tourisme d'une ville historique : l'exemple de Rouen
}

Sylvine Pickel-Chevalier

\section{(2) OpenEdition}

1 Journals

Édition électronique

URL : http://journals.openedition.org/tourisme/558

DOI : 10.4000/tourisme.558

ISSN : 2492-7503

Éditeur

Éditions touristiques européennes

\section{Édition imprimée}

Date de publication : 1 décembre 2012

Pagination : 46-60

ISSN : 2109-5671

\section{Référence électronique}

Sylvine Pickel-Chevalier, « Les processus de mise en tourisme d'une ville historique : l'exemple de Rouen », Mondes du Tourisme [En ligne], 6 | 2012, mis en ligne le 30 septembre 2015, consulté le 19 avril 2019. URL : http://journals.openedition.org/tourisme/558 ; DOI : 10.4000/tourisme.558

\section{cc) (†) $\ominus$}

Mondes du tourisme est mis à disposition selon les termes de la licence Creative Commons Attribution - Pas d'Utilisation Commerciale - Pas de Modification 4.0 International. 


\title{
Les processus de mise en tourisme
}

\section{d'une ville historique : l'exemple de Rouen}

\author{
SYlVine PiCKel-CheVAlier
}

[sylvine.chevalier@univ-angers.fr]

Géographe, maître de conférences, ITBS (université d'Angers)

Résumé. La longévité de la dynamique touristique, parfois deux fois séculaire, de certaines villes historiques interpelle. On peut se demander comment le tourisme s'inscrit durablement, au sens premier du terme, dans des cités anciennement constituées et comment il participe à leurs transformations contemporaines. Quels sont les processus, mais aussi les agents et facteurs de l'intégration de l'activité touristique dans une ville historique polyvalente (ne vivant pas exclusivement du tourisme) ? La ville médiévale de Rouen, bénéficiant d'une fréquentation touristique née au XIXe siècle et qui ne semble pas s'essouffler à l'aube du XXl', offre une étude de cas intéressante à observer. Dans ce cadre, on analysera les processus de mise en tourisme de la ville, qui évoluent avec les centres d'intérêt de la société, et participent à la définition dynamique de l'image de Rouen, mais aussi à son aménagement. En effet, ces processus contribuent, par les créations nouvelles qu'ils engendrent en termes de représentation et d'utilisation des lieux, à une réinvention permanente de la ville. Ils émanent des choix et sélections des touristes eux-mêmes, certes, mais aussi de la volonté des acteurs locaux de renforcer et de pérenniser les flux. Ces derniers n'en demeurent pas moins partiellement spontanés ou inattendus, en fonction des besoins et aspirations en constante mutation des vacanciers.

Abstract. The longevity of the touristic dynamic of the historic cities, sometimes twice secular, call out questions. We can wonder how tourism get involved into old cities and participate to their contemporary evolutions. Which are the processes, but also the agents and factors of the integration of the touristic activity into an historic and polyvalent city (living on several activities, including tourism)? The medieval town of Rouen, that has received tourists since the $19^{\text {th }}$ century till todays, is an interesting case to study. It will give us the possibility to analyze the processes of touristification of the city, that change with the centers of interests of the society, and participate to the dynamic definition of the image of Rouen, as to its development. They contribute, through the new representations and uses of the places that they create, to a constant reinvention of the city. Those processes emanate from the choices and selections of the tourists themselves, but also of the will of the local actors to strengthen and to perpetuate the touristic flows. Nonetheless, those ones are still partially spontaneous or unexpected, in function of the needs and desires in constant evolution of the tourists. 
$\mathbf{L}^{\prime}$ attractivité touristique de la ville n'est pas un phénomène récent, malgré sa prise de conscience tardive dans les sphères politiques, au soir du $\mathrm{XX}^{\mathrm{e}}$ siècle (Violier et Zarate 2007). Elle est contemporaine de la genèse des pratiques touristiques au XIX ${ }^{\mathrm{e}}$ siècle : les cités historiques d'Europe ou du Proche-Orient constituaient déjà autant d'étapes ou de destinations des premiers déplacements d'agrément $^{(1)}$. Or, cet intérêt ne s'est pas atténué au cours des siècles, et certaines villes ont conservé leur polarité touristique jusqu'à nos jours. La longévité de cette attractivité, alors même que les villes et les centres d'intérêt ont évolué, interpelle. On peut se demander comment le tourisme s'intègre "durablement", au sens premier du terme, dans une cité déjà anciennement constituée, et comment il participe à ses transformations contemporaines. Quels sont les processus, mais aussi les agents et facteurs de l'intégration de l'activité touristique dans une ville historique ? Dans ce cadre, on s'intéressera plus particulièrement à l'étude de Rouen. Le chef-lieu de la région de Haute-Normandie et du département de la Seine-Maritime jouit d'une longue attractivité touristique, née au xixe siècle et qui perdure jusqu'à aujourd'hui. En effet, cette ville, qui compte 110927 habitants (525 638 habitants avec sa communauté d'agglomération, ce qui représente la $14^{\mathrm{e}}$ aire urbaine de France), bénéficie en 2010 d'une augmentation de la fréquentation touristique de ses grands événements et animations (la fréquentation de “Rouen sur mer" a été multipliée par 2,2 entre 2009 et 2010), mais aussi de ses hôtels (35\% de plus par rapport à $\left.2009^{(2)}\right)$. Le tourisme, anciennement inscrit dans la ville, ne semble donc pas en proie à l'essoufflement.

En cela, elle nous paraît être une étude de cas intéressante à analyser. En effet, on peut s'interroger sur les processus d'intégration du tourisme à Rouen, qui constitue une ville touristique (ville polyvalente) et non une ville "touristifiée" (Équipe Mit, 2002, 2005). Afin de comprendre ce phénomène, on mènera une analyse qui repose sur l'étude de différentes sources, bibliographiques, iconographiques et statistiques, mais aussi sur l'exploitation de rapports d'activité. Nous utiliserons de même une enquête réalisée sur les publics fréquentant Rouen sur mer ${ }^{(3)}$, ainsi que des entretiens menés auprès de représentants de la collectivité territoriale, qui nous permettront d'étudier les discours officiels et les axes défendus par les décideurs politiques.

Les analyses conduites à partir du croisement de ces différentes données nous permettront d'essayer de mieux saisir les facteurs de la mise en tourisme pérenne de Rouen. Dans ce contexte, nous étudierons en premier lieu les processus de "co-constitution" entre mise en tourisme et valorisation du patrimoine de la ville. Nous nous consacrerons, en second lieu, aux efforts des acteurs locaux pour éviter le phénomène de muséification qu'ils pourraient impliquer. Dans ce cadre, on analysera les politiques globales d'intégration du tourisme dans la ville, qui participent intrinsèquement aux évolutions de son identité, par les transformations de l'aménagement des lieux, mais aussi de leurs pratiques et représentations. Et ce, à destination des touristes, mais aussi des résidents permanents qui se réapproprient leur ville, mise en scène et valorisée pour les visiteurs.

\section{UNE VILLE HISTORIQUE REDÉFINIE PAR \\ SA MISE EN TOURISME : \\ LE PHÉNOMÈNE DE "CO-CONSTITUTION"}

L'attractivité touristique urbaine émane d'une complémentarité de l'offre, reposant essentiellement sur la redécouverte du patrimoine, le goût pour la modernité, qui s'exprime notamment dans les grandes réalisations architecturales et urbanistiques, et la participation à de grands événements (Duhamel et Knafou, 2007). À cela, on peut ajouter l'accessibilité et la diversité des services, contribuant à la centralité de la ville, parmi lesquels l'offre “shopping" s'octroie une importance prépondérante $^{(4)}$. Or, cette association d'attraits caractérise Rouen.

\section{Un patrimoine reconnu : le rôle du regard extérieur}

Rouen, dressée le long de la Seine à 130 kilomètres de Paris, est une ville du Moyen Âge qui se démarque par la diversité de son bâti. En effet, dans son quartier historique s'égrainent des maisons symbolisant la tra- 
Schéma | - Les déséquilibres

de la ville historique et touristique de Rouen

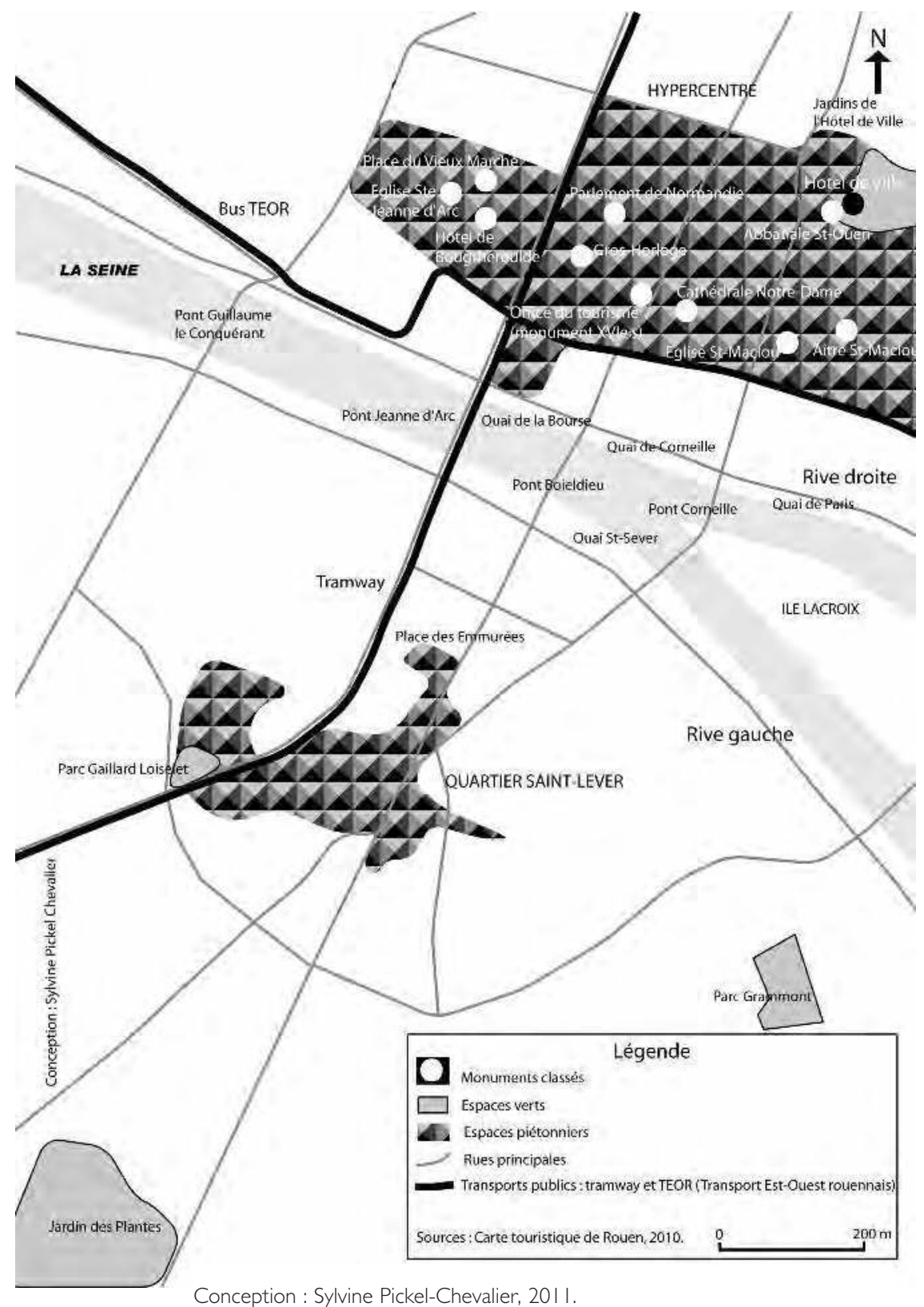

versée des siècles de la ville, depuis le XII siècle jusqu'à l'époque contemporaine. Elle est riche de 227 maisons et monuments inscrits ou classés par les monuments historiques (dont 16 églises, chapelles et cathédrale, le parlement de Normandie, le Bureau des finances devenu office du tourisme, l'hôtel de Bourgtheroulde, le Gros-Horloge, 1' "aître SaintMaclou”, le jardin des plantes, des théâtres, des portes et remparts, des $\operatorname{parcs}^{(5)} \ldots$.. (cf. schéma 1$)$, ce qui place Rouen parmi les six premières villes de France en termes de richesse patrimoniale. Cette profusion d'habitations et de monuments de différentes époques témoignent de la réussite économique pluriséculaire de la ville, qui provient notamment de sa situation géographique sur le fleuve, aux portes de la capitale. Rouen bénéficie aussi de la présence de 2000 maisons à pans de bois, héritées du Moyen Âge et reconnaissables à leur structure en encorbellement (surtout concentrées dans la rue du GrosHorloge, la rue Saint-Romain et autour de la place du Vieux-Marché où fut brûlée Jeanne d'Arc), mais aussi de l'époque moderne et du XIX ${ }^{e}$ siècle, pour les plus récentes. Monuments classés et maisons à pans de bois se concentrent dans des quartiers du centre-ville protégés depuis 1964 par un secteur sauvegardé - 36 hectares autour de la cathédrale -, dans le cadre de la loi Malraux. À l'égal de Lyon et de Sarlat, Rouen fait ainsi partie des premières villes à en avoir profité.

Cette protection officielle témoigne de l'intérêt d'ores et déjà accordé au bâti de la ville, intérêt qui, en effet, a vu le jour dès le XIX siècle, alors qu'émergeait en France la notion de patrimoine (Babelon et Chastel, 2000). Or, celle-ci relève d'un regard extérieur à même de comparer et de sélectionner les objets de la mémoire collective. Ce fut certes le rôle de l'inspecteur des monuments historiques à partir de 1830, mais il ne faut pas sous-estimer l'importance des touristes, qui furent aussi des acteurs de la reconnaissance et de la diffusion des lieux, devenant 
héritage commun de la nation. La corrélation entre valorisation du patrimoine et attractivité touristique, qui permet de parler de phénomène de co-constitution, n'est plus à démontrer (Lazzarotti et Violier, 2007).

\section{Artistes et touristes, créateurs de l'image de Rouen}

La mise en place précoce de politiques de protection du patrimoine de Rouen témoigne de l'ancienneté de l'intérêt qu'on lui prête. Ce phénomène s'explique notamment par l'accessibilité de la ville depuis la région parisienne, important bassin émetteur de touristes, mais aussi par la richesse du bâti médiéval. Celuici séduit particulièrement les romantiques, notamment grâce à l'œuvre de Victor Hugo, qui a largement concouru à sa revalorisation, après des siècles de dénigrement (Sire, 2005). Le célèbre auteur a d'ailleurs contribué à la codification touristique et patrimoniale de Rouen, écrivant en 1831: "Amis! C'est donc Rouen, la ville aux vieilles rues, Aux vieilles tours, débris des races disparues - La ville aux cent clochers carillonnant dans l'air, - Le Rouen des châteaux, des hôtels, des bastilles, - Dont le front hérissé de flèches et d'aiguilles - Déchire incessamment les brumes de la mer." ("Feuilles d'automne".)

En ces quelques vers, qui reprennent les schèmes de la sensibilité romantique (nostalgie par rapport aux époques révolues; valorisation du patrimoine médiéval ; célébration de l'océan même lointain ; champ lexical du sublime gravitant autour

|llustration I • Turner : La Cathédrale de Rouen ( I 832)

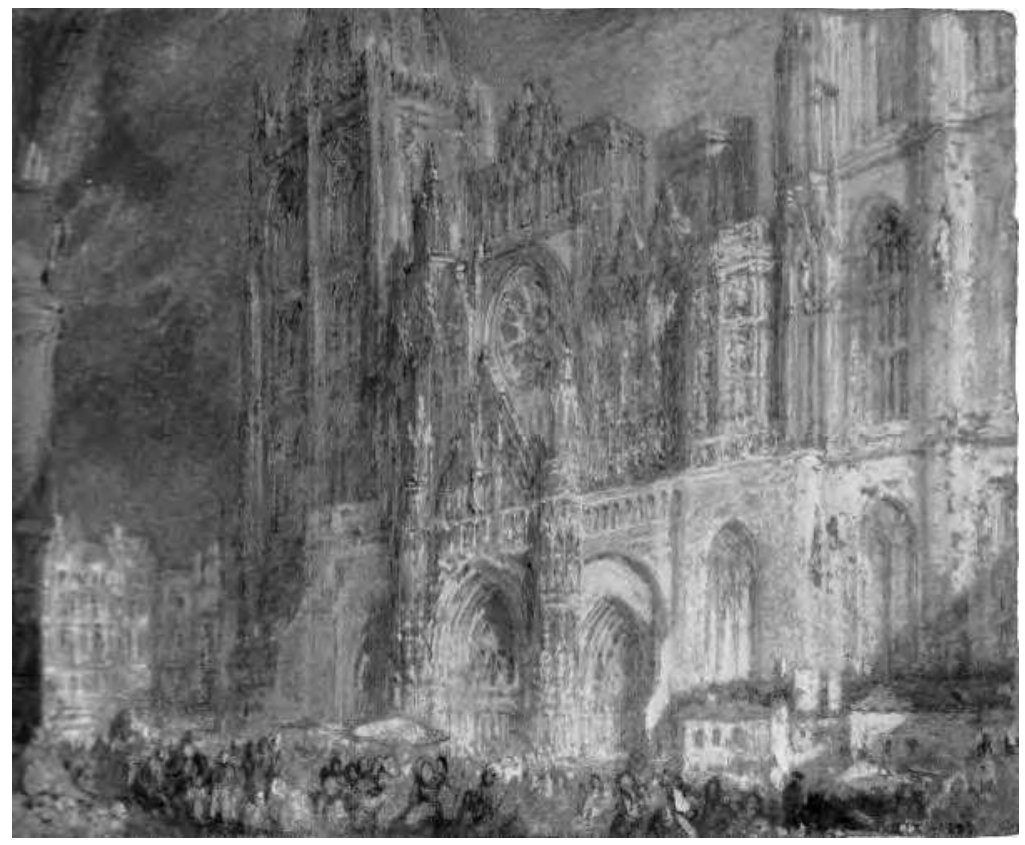

Source : Salomé (2010, p. 189).

de la terreur délicieuse), s'amorce la construction de l'image de la ville, au travers de ses objets patrimoniaux. Elle se poursuit avec l'œuvre de Stendhal, qui fait de Rouen une destination de son itinéraire touristique, qu'il relate en 1837 dans Mémoires d'un touriste. Et ce, notamment pour la diversité de ses maisons médiévales dont les murs "sont formés par de grands morceaux de bois", mais plus encore pour la richesse de ses églises, qui lui font assimiler la ville à "l'Athènes du genre gothique". Il est intéressant de souligner que Stendhal aurait élaboré cette description de Rouen comme un guide, afin de répondre à la demande de Mérimée, alors Iinspecteur des monuments historiques, en reprenant ce qui était déjà particulièrement prisé dans la ville. Une fois encore, on observe les rela- tions intrinsèques associant valorisation du patrimoine et attractivité touristique. Cette fascination des romantiques s'exprime aussi dans la peinture, au travers de l'œuvre d'artistes nationaux et internationaux. Ainsi, Joseph William Turner, Johan Barthold Jongkind ou encore Paul Huet consacrent plusieurs toiles à des vues de la ville - Rouen, vers l'amont (1832); La Cathédrale de Rouen (1832) (cf. illustration 1) ; Rouen, avec la côte Sainte-Catherine (1858) ; Vue générale de Rouen, prise du Mont-aux-Malades (1831).

CEuvres littéraires, récits de touristes - qui parfois se confondent et peintures sont donc fondamentales dans la construction de l'image de la ville, car elles contribuent à forger et à véhiculer ses représentations, autour de la reconnaissance de quelques objets choisis pour l'iden- 
Illustration 2 - Pissarro

Pont Boieldieu à Rouen, temps mouillé (1896)

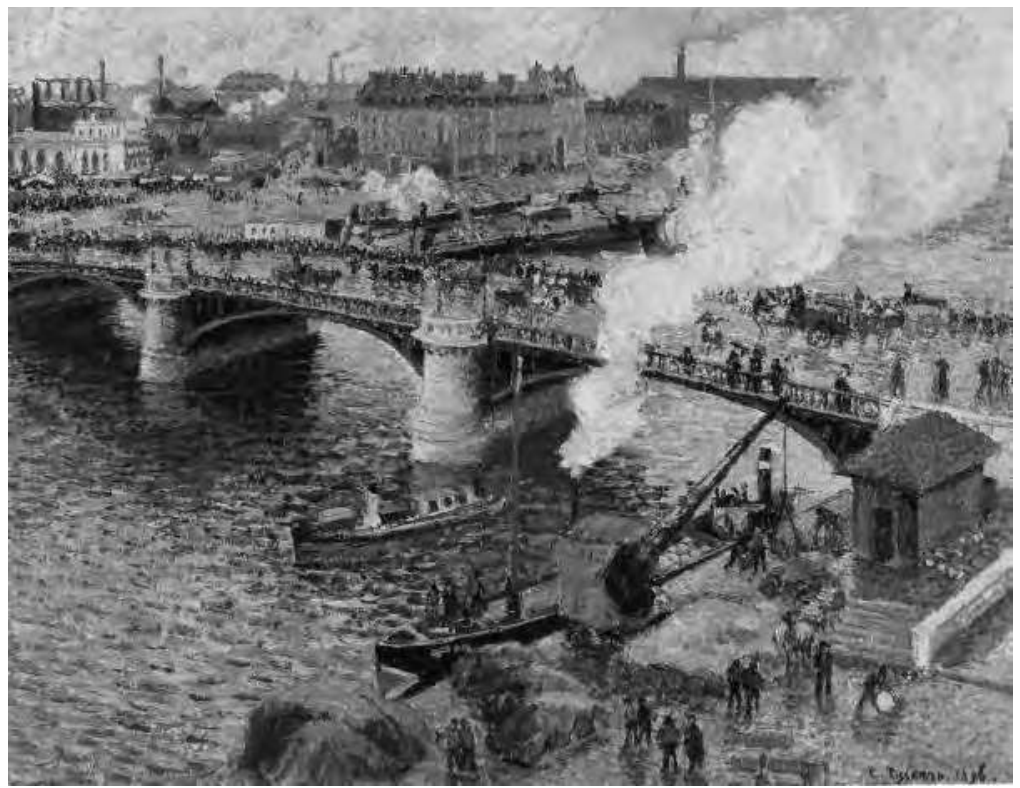

Source : Salomé (20 10, p. 290).

tifier - la cathédrale, les rues médiévales, les bords de Seine....

Ce phénomène n'est pas propre à Rouen (Bertho-Lavenir, 1999), mais il s'y exprime particulièrement. Les codes définissant la ville dès 1830 au travers de son patrimoine devenant touristique ne sont pas remis en cause à la fin du siècle, lorsque la Belle Époque succède au XIX ${ }^{e}$ siècle, et que le romantisme laisse place à l'impressionnisme. Loin de se détourner des centres d'intérêt de leurs prédécesseurs, les artistes de ce courant nouveau investissent à leur tour la ville. Boudin, mais surtout Pissarro, Gauguin et Monet lui ont dédié de nombreuses œuvres, célébrant eux aussi les monuments, les rues médiévales animées, les bords de Seine (Salomé, 20I0). Monnet s'illustre particulièrement en immortalisant sur vingt toiles, exposées en 1895, les différentes lumières du jour sur la cathédrale gothique flamboyante. Les impressionnistes reprennent, de la sorte, les schèmes déjà établis, mais ils remplacent la mélancolie de leurs aïeux par l'enthousiasme de leur époque. La croyance dans les prouesses de la modernité et les promesses de l'avenir s'exprime dans la valorisation aussi des lieux industriels et animés, tels que le pont Corneille ou le pont Boieldieu, qui deviennent désormais, par les œuvres de Pissarro notamment (cf. illustration 2), des composantes participant à l'identification de la ville. Enfin, Rouen fut aussi un berceau du mouvement post-impressionniste, qui porte le nom d'école de Rouen et rassemble, entre autres, Angrand, Lemaître, Frechon, Dubois-Pillet, Delattre et, après eux, Lebourg,
Pinchon, Denis, Laprade... Ces derniers se sont aussi attaché à immortaliser l'atmosphère changeante et brumeuse de la cité industrielle des bords de Seine, en pleine effervescence (Salomé, 20 I0).

L'intérêt pour le patrimoine de Rouen ne s'est pas démenti pendant l'entre-deux-guerres, ni au sortir de la Seconde Guerre mondiale, comme en témoignent les importants aménagements du centre-ville. Afin de pallier la dévastation par les bombardements, de plus de 100 hectares dans les quartiers anciens, une politique de reconstruction fut adoptée, en respectant les tracés des rues anciennes sur la rive droite - des constructions plus modernes furent réalisées rive gauche. La valorisation du patrimoine rouennais fut encore renforcée par l'affirmation des politiques de protection et notamment la mise en place, évoquée plus haut, en 1964, d'un secteur sauvegardé de 36 hectares autour de la cathédrale. À l'aube du XXI siècle, cette volonté de préservation ne s'est pas essoufflée, et bénéficie d'un important renouveau, alors que la dynamique touristique est plus que jamais consciemment associée à celle de la valorisation du patrimoine (Lazzarotti et Violier, 2007).

\section{Des conditions indispensables}

à la fréquentation touristique : accessibilité et centralité

La forte fréquentation de Rouen s'explique aussi par son accessibilité et sa centralité, essentielles à la définition de la ville (Boullier, 2010). Rouen jouit d'une situation privi- 
légiée par son statut de capitale d'une région très touristique - Haute et Basse-Normandie occupent ensemble la $8^{e}$ place des régions touristiques françaises, en accueillant $5,1 \%$ des séjours et 3,9\% des nuitées (Observatoire régional de tourisme Normandie, 2006) - mais aussi par sa localisation à proximité de Paris. Elle est située à 130 kilomètres de la capitale, à laquelle elle est reliée par l'autoroute, les routes nationales devenues départementales (déclassement en 2006) et le chemin de fer (depuis 1841). Cette proximité de Paris est à la fois un atout et un inconvénient. En effet, elle favorise la fréquentation touristique par ce qui constitue le premier bassin de population de France, mais elle bride aussi la centralité rouennaise, en en limitant l'influence. Néanmoins, cette dernière demeure importante à l'échelle de la région. Grâce à quelque 3000 magasins, ayant un ciblage très large, depuis les produits d'entrée de gamme jusqu'aux produits de luxe, 350 restaurants et brasseries, 60 hôtels dominés par les 2 et 3 次, mais allant jusqu'au 5 不 (hôtel de Bourgtheroulde), Rouen constitue le $8^{\mathrm{e}}$ pôle commercial à ciel ouvert de France. Cette profusion favorise la venue de la population locale, certes, mais aussi des touristes et excursionnistes. Et ce, d'autant que les magasins proposent, en plus de la variété des gammes, une pluralité de styles, et que se juxtaposent enseignes célèbres (Printemps, Zara, Pimkie, Timberland...) et magasins indépendants originaux, voire insolites, notamment dans la bijouterie, les arts et les produits de bouche. Cette association du connu et de l'inconnu, qui contribue à définir la ville par le couple densité-diversité (Lévy, 2003, p. 99), est aussi une composante essentielle de l'attractivité touristique (Équipe Mit, 2002).

Ainsi, on comprend mieux les processus de co-développement touristique et patrimonial de la ville de Rouen, à l'œuvre depuis le XIX siècle. Néanmoins, la continuité de sa fréquentation au XXI ${ }^{e}$ siècle suppose d'autres composantes, qui permettent de poursuivre la valorisation de la ville historique, tout en renouvelant son image. Dans ce contexte, l'événementiel s'octroie un rôle essentiel (Duhamel et Knafou, 2007), comme l'exploitation de l'art contemporain qui permet de compenser les représentations "passéistes" d'une ville patrimonialisée.

\section{L'ÉVÉNEMENTIEL ET L'ART CONTEMPORAIN, POUR ÉCHAPPER \\ AU PROCESSUS \\ DE SANCTUARISATION}

Le patrimoine bâti, mis en tourisme depuis le XIX ${ }^{e}$ siècle et associé au foisonnement de l'offre en termes d'hébergement, de restauration et de commerces, constitue donc le socle initial sur lequel s'est construite la ville touristique de Rouen. Toutefois, cette richesse patrimoniale peut constituer aussi un obstacle à sa fréquentation au XXI ${ }^{\mathrm{e}}$ siècle, en enfermant la ville dans une image passéiste ou en l'entraînant dans un processus de muséification.
Conscients de ce danger, les acteurs politiques locaux ont tablé sur la mise en place de nombreux événements, destinés à inscrire la ville dans son présent. Cette volonté n'est pas exceptionnelle. Selon Christine Laroche et Philippe Hermet, elle caractérise un certain nombre de villes historiques, que les décideurs refusent de sanctuariser comme un "objet admirable, mais passé et fiché", pour les associer à "un projet innovant, contradictoire, en mouvement" (20।0). Dans ce cadre, on observe à Rouen la recherche affirmée des acteurs de la ville de valoriser les événements qu'ils disent "décalés" "(), c'est-à-dire inattendus, paradoxaux dans le contexte local, afin de contribuer par l'insolite à cette dynamique.

\section{Une programmation \\ d'“événementiels", pour}

dynamiser la ville toute l'année

Cette volonté s'exprime particulièrement fortement depuis l'arrivée de la nouvelle municipalité socialiste à la tête de la ville, en 2008. Elle a mis en place une programmation saisonnière de l'événementiel à Rouen, destinée à la rendre attractive toute l'année. Ainsi, se succèdent : "Rouen givré", fin novembre-début janvier (patinoire, village d'enfants, illuminations, marchés de Noël) ; "Printemps de Rouen", d'avril à mai (événements musicaux, consistant dans l'organisation de quatrevingt-dix concerts dans les lieux publics de l' "hypercentre", et gratuité de tous les musées municipaux de la ville pendant les vacances de 


\section{Illustration $3 \cdot$ Plage de Rouen sur mer, accessible par le pont Boieldieu, devenu piétonnier et orné de la sculpture monumentale Camille}

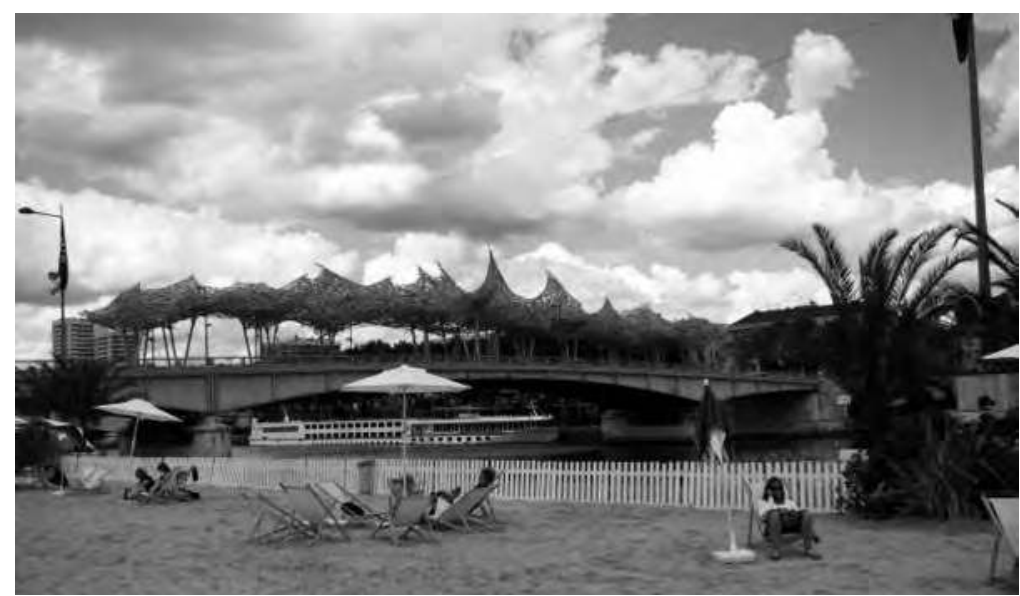

Photo : Sylvine Pickel-Chevalier, été 2010.

printemps) ; "Rouen sur mer", de juin à août (fabrication d'une plage proposant de nombreuses activités gratuites) ; et "Rouen impressionné", de septembre à octobre (événement dédié à la célébration de l'art, mettant en parallèle impressionnisme et art contemporain). À cela s'ajoute toujours, tous les quatre ans, l'organisation de l'“Armada". Afin de comprendre davantage les processus, nous nous focaliserons sur les événements qui drainent le plus de visiteurs, à savoir l'Armada, Rouen sur Mer et Rouen impressionné. Il est intéressant d'observer que la politique des acteurs de la ville n'est pas de s'affranchir de la richesse patrimoniale de Rouen, mais de s'en servir comme d'un tremplin à la modernité, en associant le plus souvent héritage et nouveautés.

Dans ce contexte, s'illustre l'Armada, lancée en 1989 pour célébrer le bicentenaire de la Révolution française et réitérée depuis, tous les quatre ou cinq ans, en début d'été. Cet événement, qui attire des flux touristiques au nombre estimé de 400000 à 600000 personnes par jour pendant dix jours ${ }^{(7)}$, se démarque pour associer vieux gréements, bateaux de guerre fleurons de la modernité navale, mais aussi péniches de très grande taille. L'idée est de valoriser la dynamique maritime de la ville, en ne se cantonnant pas à son passé révolu. Afin d'optimiser cette attractivité touristique internationale, la municipalité a entrepris, depuis 2008, d'enrichir l'Armada (organisée par une association) par la mise en place d'événements festifs associés (bals, défilés des équipages, fanfares...), qui permettent de drainer les visiteurs sillonnant les quais vers le centre-ville. Si le chiffre d'affaires exact des commerçants pendant l'événement n'est pas connu, le nombre de demandes en matière de prestations commercialisées sur la ville auprès de l'office du tourisme a progressé du double au quintuple, en fonction des activités, par rapport à l'édition précédente ${ }^{(8)}$.

L'été rouennais est aussi dynamisé par l'organisation, depuis 2009, de Rouen sur mer. Le projet s'est bien sûr inspiré de l'initiative de Bertrand Delanoë qui, de façon non intentionnelle, a créé en 2002 un nouveau centre d'intérêt touristique dans la capitale, en fermant ses quais l'été à la circulation pour y aménager un espace ludique associant plage et animations. Ce projet, initialement destiné à distraire les Parisiens ne pouvant partir à la mer, a rapidement crée l'engouement des populations touristiques, venant voir l'événement et y participer euxmêmes. L'événement à Rouen, qui consiste en l'aménagement de 18000 $\mathrm{m}^{3}$ de sable (2010) sur les quais bas de la rive gauche - notamment quai Saint-Sever ( $c f$. illustration 3) - et dans l'organisation d'une trentaine d'animations et activités gratuites (jeux pour enfants, châteaux gonflables, terrains de sport, fosse de plongée, espace de bibliothèque... ), nourrit le même dessein initial économique et social. En effet, l'objectif est avant tout de répondre aux besoins des populations modestes ne pouvant partir en vacances et de redynamiser la rive gauche de la ville, qui souffre d'un ralentissement économique.

Cette initiative est un succès, comme l'atteste sa fréquentation par 15000 personnes en 2010, contre 68000 en 2009. Elle est plébiscitée par la population locale de 
la rive gauche $(28 \%$ de la fréquentation) comme de la rive droite (22\%), mais aussi par les touristes, qui représenteraient $45 \%$ des visiteurs, selon une enquête menée par la ville en 2010 (on regrettera toutefois de ne pas en savoir plus sur l'échantillon interrogé, notamment le nombre de participants à l'enquête $\left.{ }^{(9)}\right)$. La réussite de Rouen sur mer s'explique aussi par son intégration dans une politique plus globale de dynamisation de la ville, associant d'autres événements, comme "Terrasses du jeudi" $\left(10^{\mathrm{e}}\right.$ édition en 2010), qui consiste en l'organisation de trente concerts de tout type sur les terrasses des cafés du centre-ville. Leur combinaison leur est mutuellement profitable, comme en témoigne l'essor de la fréquentation des concerts depuis la création de Rouen sur mer en 2009 (passage de 35000 visiteurs en 2008 à 65000 en 2010).

Ce phénomène permet de revenir sur un élément fondamental pour la compréhension de la réussite touristique de la ville historique, à savoir la pluralité de ses centres d'intérêt qui en font une destination touristique (Violier, 2009), associant les choses à voir et à faire. De plus, comme le souligne Rémy Knafou, “aujourd'hui, il n'est plus possible de songer à animer la ville pour ses seuls habitants ou bien à concevoir des attractions pour les seuls touristes” (2007, p. 12). Les deux populations se fréquentent sur les mêmes espaces en pratiquant, certes, le plus souvent des activités différentes, mais pas toujours. Ainsi, week-ends et fins de journée sont l'occasion pour les populations résidentes d'habiter autrement leur ville, en flânant ou en se livrant aux mêmes pratiques que les touristes (Lazzarotti et Pickel, 2008).

Enfin, les acteurs de la ville aspirent à inscrire encore davantage Rouen dans son présent, voire son avenir, en exploitant le registre de l'art contemporain. Ce dernier est censé faire écho au patrimoine bâti et aux arts plus anciens (romantisme, impressionnisme), en empêchant l'enfermement de la ville dans sa richesse culturelle passée.

\section{L'art contemporain, vecteur de redynamisation d'une ville historique?}

La municipalité de Rouen a exploité l'organisation de "Normandie impressionniste", mis en place par les Haute et BasseNormandie en 2010, pour mettre la ville en valeur. Et ce, en associant le patrimoine traditionnel local à des expositions dédiées à l'art contemporain, au sein de l'événement Rouen impressionné. L'idée des décideurs politiques est d'utiliser le symbole de modernité porté par les impressionnistes, annonçant l'entrée dans le xxe siècle, pour valoriser des œuvres contemporaines, disséminées dans la ville. Ainsi, alors que l'événement Normandie impressionniste s'est traduit à Rouen par l'exposition "Une ville pour l'impressionnisme : Monet, Pissarro et Gauguin à Rouen”, rassemblant une centaine de toiles au musée des Beaux-Arts (qui a accueilli 182932 visiteurs français et étrangers entre juin et septembre), Rouen impressionné a mis en avant des artistes renommés contemporains, en leur offrant la ville comme terrain d'expression. L'artiste belge Arne Quinze a mis en valeur le pont Boieldieu, prisé par les peintres depuis le XIX ${ }^{e}$ siècle, en y édifiant une sculpture monumentale aux couleurs vives, intitulée Camille. Le dessein en était l'appropriation du pont piétonnier pendant l'été, permettant d'intégrer davantage la rive gauche au reste de la ville. Cette initiative fut un succès, puisque le pont a largement été utilisé, notamment pour accéder à Rouen sur mer, auquel il donne directement accès ( $c f$. illustration 3 ). L'artiste a aussi investi l'abbatiale classée de Saint-Ouen avec une exposition de toiles inspirées des Nymphéas, nommée "Les Jardins" (cf. illustration 4). L'objectif était d'amener des visiteurs peu sensibilisés à l'art contemporain à le découvrir, dans le cadre d'un site gothique classé. Or, cette initiative fut une réussite, puisque l'abbatiale a multiplié sa fréquentation par près de deux entre l'été 2009 (10 616) et l'été 2010 (20 550 visiteurs $)^{(10)}$. Cela signifie que l'insolite de la rencontre entre l'art gothique et l'art contemporain a favorisé la découverte du second certes, mais a aussi permis de régénérer l'intérêt pour le premier.

D'autres événements mettant à l'honneur la modernité et l'innovation ont été organisés, comme l'exposition “Appel d'air" de la Japonaise Shigelo Hirakawa, au jardin des plantes, célébrant la photo- 


\section{Illustration 4 - Exposition "Les Jardins" dans l'abbatiale Saint-Ouen}

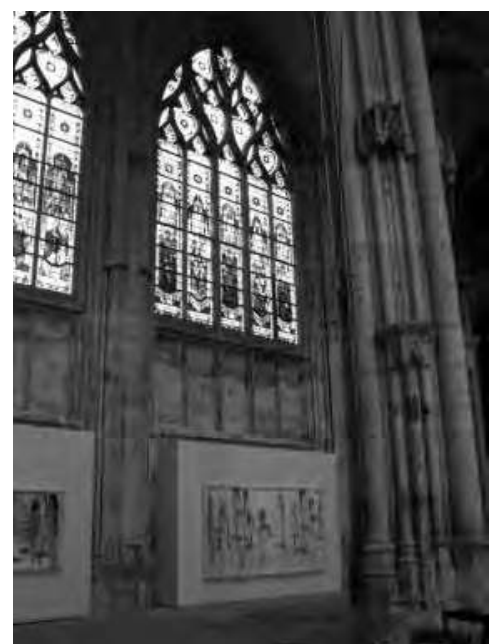

Photo : Sylvine Pickel-Chevalier, été 2010.

synthèse et l'air (association d'arbres et de molécules d'oxygène) ; ou encore la mise en place de ruches urbaines en centre-ville, modélisées par l'artiste-apiculteur Olivier Darné. Enfin, la place de l'hôtel de ville a aussi été animée tout l'été par les Cubes de lumière, de Jérôme Toq'R, œuvre peinte intergénérationnelle et participative (les visiteurs étaient invités à peindre eux-mêmes les compositions). Il est intéressant d'observer que ces différents événements ont été disséminés sur les deux rives du centre de Rouen, afin de ne pas renforcer les déséquilibres perdurant au bénéfice de la rive droite, qui concentre le vieux centre historique piétonnier et la majorité des monuments classés ( $c f$. schéma 1$)$.

Ainsi, la réussite touristique de la ville historique de Rouen émane bien de la diversité de l'offre, mais aussi de cet écho entre patrimoine classé, innovation, et renouvellement apporté par l'événementiel qui, selon le sociologue Dominique Boullier, dote la ville d' "une capacité étonnante de réinvention d'elle-même” (2010, p. 93). Il est intéressant de souligner, par ailleurs, que cette combinaison de la valorisation des marqueurs de l'histoire et des symboles du présent, voire du futur, n'est pas propre aux villes touristiques du $\mathrm{XIX}^{\mathrm{e}}$ siècle, mais elle les caractérise depuis le XIX ${ }^{\mathrm{e}}$. En effet, comme en témoignent les œuvres impressionnistes de Rouen, l'intérêt prêté aux lieux provenait déjà de la rencontre du patrimoine culturel (patrimoine bâti) et naturel (Seine), ainsi que de l'effervescence de la révolution industrielle (rues animées, chemin de fer, innombrables cheminées d'usines ou de bateaux à vapeur, déversant leur fumée dans le gris du ciel - voir les illustrations 1 et 2). Cette fascination pour la confrontation entre les vestiges du passé et les promesses de l'avenir caractérise de fait la sensibilité du
XIX $^{e}$ siècle, avant même la Belle Époque, comme le rappellent les écrits et peintures des romantiques (Victor Hugo, Turner).

On comprend ainsi davantage le fonctionnement de la ville historique de Rouen, relevant d'une mise en tourisme associant patrimonialisation et dynamisme contemporain. Ce dernier repose notamment sur la valorisation de l'art contemporain et sur l'événementiel, qui symbolise, par son caractère éphémère, un constant renouvellement. Ce processus repose donc sur une volonté essentielle des décideurs, qui ne se limitent toutefois pas à ces seules animations, porteuses de l'image de la ville. En effet, ils intègrent de façon plus intrinsèque le tourisme à l'évolution de la ville historique, en l'agrégeant dans les politiques globales d'aménagement, de transports et de communication.

\section{POUR ORCHESTRER}

LE TOURISME DANS

LA VILLE HISTORIQUE :

UNE POLITIQUE GLOBALE

D'INTÉGRATION

\section{Structurer la ville historique et touristique : \\ aménagement et transport}

Si le tourisme est urbain depuis sa genèse, en se situant en amont et en aval du processus ${ }^{(11)}$, la reconnaissance de ce fait auprès des collectivités locales est plus récente. En effet, il faudra attendre les années 1990 pour assister à l'émergence d'une réelle prise de conscience des politiques, quant à l'importance de 
la dynamique touristique dans l'espace urbain. Et ce, aussi bien en tant que moteur économique que composante à part entière de l'image et $\mathrm{du}$ fonctionnement de cet espace (Violier et Zarate, 2007). Ce phénomène est d'autant plus fort dans les villes historiques, dont l'aménagement doit supporter les objectifs parfois opposés de conservation et de progrès. Ces politiques devant permettre de concilier intérêt des visiteurs et des Rouennais passent par l'aménagement. Celui-ci constitue un outil fondamental de "l'habiter" de la ville, à l'heure où les distinctions entre espaces et usages touristiques et résidentiels s'estompent. En effet, les populations locales apprennent à "habiter touristiquement" leur ville (Stock, 2007), c'està-dire à la vivre de façon non utilitaire, à déambuler pour profiter de ses attraits esthétiques et ludiques. Cette situation est observable à Rouen, alors que les populations touristiques et résidentes se mêlent dans les sites attractifs (hypercentre associant patrimoine bâti et commerces) et les événements polarisants (Armada, Normandie impressionniste, Rouen impressionné, Rouen sur mer).

Certains lieux deviennent donc des lieux polyvalents, utilisés par les visiteurs comme par les populations résidentes (Laroche et Hermet, 2010). Loin de ne relever que de phénomènes spontanés, cette évolution est largement orchestrée par les politiques locales d'aménagement. Ainsi, dans le but de favoriser l'attractivité de l'hypercentre commer- çant, la municipalité l'a rendu piétonnier dès les années 1970. De même, sur une idée du maire centriste Jean Lecanuet (maire de Rouen de 1968 à 1993), l'agglomération fut dotée, un an après son départ, d'un tramway, localement appelé "le métro" en raison du fait qu'une partie de son itinéraire est souterraine (5 stations sur 23). Rouen est l'une des plus petites agglomérations de France a être pourvue d'un transport en commun en partie souterrain, ce qui témoigne une fois encore de cette volonté de l'inscrire dans le présent et l'avenir. On soulignera aussi le paradoxe du tramway, dont l'image est profondément associée aux évolutions urbaines. Considéré comme un symbole d'obsolescence, il a été démantelé dans les villes françaises dans les années 1950 (1953 pour la dernière ligne à Rouen) pour laisser place à la modernité automobile. À l'aube du XXI siècle, il redevient un marqueur de la cité moderne, alors que la voiture polluante est décriée... Dans ce contexte, on remarque la précocité de la démarche rouennaise, qui s'engage dans cet aménagement, très onéreux, dès les années 1990.

Aujourd'hui il existe aussi à un vaste projet de réaménagement de la rive gauche qui, en dépit de sa proximité géographique du centre historique, souffre toujours d'un manque de dynamisme par rapport à la rive droite. L'objectif est de parvenir à revaloriser ces quartiers délaissés et à les réintégrer dans une politique globale de "réappropriation de l'axe Seine", menée depuis 1999. Elle consiste en d'importants travaux d'embellissement et d'améliorations urbaines, notamment par la requalification des quais et de la place des Emmurées, lieu emblématique du quartier Saint-Sever. Le projet intègre aussi un perfectionnement des transports en commun, avec, entre autres, la reconfiguration des stations du tramway à SaintSever et la mise en service de nouvelles rames. Cette initiative a pour objectif d'atténuer les manques de la rive gauche, qui n'est pas desservie par les trois lignes du TEOR (Transport est-ouest rouennais). Ce dernier est un réseau de bus nouvelle génération, avec système de guidage et d'accostage optique, mis en service depuis 2001 ( $c f$. schéma 1).

Le rôle de l'aménagement est donc fondamental dans la mise en tourisme d'une ville historique, en lui permettant d'être accessible, mais aussi "racontable, interprétable, et donc signifiante” (Vlès, 2006, p. 1 17). Or, ce processus induit la mise en scène des espaces publics en en faisant des lieux polyvalents (quais transformés en plage ; pont devenant espace piétonnier et œuvre d'art ; centre-ville ponctué d'activités apicoles...), dont la fréquentation résulte de leur combinaison avec une politique globale des transports et des déplacements. Cette politique a également inspiré la création, en 2007, d'un système de location de vélos en libre service, dénommé Cy'clic. Proposant 250 vélos répartis dans 20 stations (12 rive droite, 1 sur l'île Lacroix et 7 rive gauche), son objectif est, selon la municipalité, de permettre "aux Rouennais et aux 
visiteurs de se déplacer en ville à vélo en toute liberté, 7 jours sur 7. Cy'clic c'est la liberté de circuler, de se déplacer, la liberté de visiter la ville à son rythme ou encore de gagner du temps ${ }^{(12)}$." On observe donc que, une fois de plus, l'aménagement a pour dessein d'améliorer "l'habiter" de la ville, aussi bien à destination des résidents permanents que des touristes.

Enfin, tous ces efforts politiques pour valoriser l'image de Rouen ville historique inscrite dans la modernité ne permettent une augmentation significative de la fréquentation touristique que par la diffusion de cette image.

\section{Diffuser l'image renouvelée d'une ville historique : \\ l'importance \\ de la communication}

Les décideurs locaux aspirent, donc, à promouvoir Rouen comme une ville à la fois historique, animée et innovante, grâce à une programmation d'événements d'envergure, mais aussi à des aménagements agrégeant intimement le tourisme aux évolutions urbaines. Cependant, cette politique ne peut être assimilée que par l'intermédiaire d'une communication à destination des touristes, qui façonne aussi, par effet collatéral, l'image de la ville auprès de ses habitants. Or, la municipalité s'est fortement investie dans ce champ, en diffusant sur supports papier et en ligne, les codifications de la ville qu'elle souhaite véhiculer : patrimoine, animations, commerces, culture, événements, convi- vialité, accessibilité.

Ce dernier aspect s'illustre notamment, dans le cadre de Rouen impressionnée, par une volonté affirmée d'introduire l'art contemporain dans les lieux polyvalents. Les œuvres, souvent peu connues du grand public, ne furent pas isolées dans des musées spécifiques, qui extraient l'art de la vie quotidienne et exigent une démarche volontariste des visiteurs. Au contraire, les compositions furent toutes localisées dans des sites fréquentés (rues du centre-ville, jardin des plantes, hôtel de ville, abbatiale Saint-Ouen, pont Boieldieu...). De même, les expositions de photos "Fujisan" et "Des rives", de François Cavelier, étaient proposées dans les Galeries Lafayette. L'objectif de cette approche est double : à savoir, changer le regard porté sur les œuvres contemporaines, souvent méconnues du grand public ; mais aussi transformer les représentations et usages des lieux urbains qui les accueillent. Cette volonté s'exprime au travers des propos de Laure DelamotteLegrand, directrice artistique de Rouen impressionnée, qui résume le dessein de l'exposition en ces termes : "L'art contemporain sera accessible à tous, dans des oeuvres transformant les sites urbains, en ceuvres à vivre et à arpenter. ${ }^{(13) ”}$

On constate par ailleurs que la référence à l'impressionnisme pour cette exposition n'est pas fortuite, ce mouvement incarnant un moment de rupture artistique, auquel les œuvres présentées veulent faire écho. Or, cette politique parfois audacieuse de mélange des genres a rencontré un vrai succès, au vu du nombre des visiteurs pour chacune des expositions : 20550 pour "Les Jardins", à l'abbaye Saint-Ouen ; 1 500, dont 600 enfants, pour les Cubes de lumière ; 1463 pour "Fujisan" et "Des rives"; 1300 pour les "Niches urbaines" ; 395 visites guidées de Camille et 375 d' "Appel d'air", auxquelles s'ajoutent des centaines de passants quotidiens.

L'objectif de cette politique est, une fois encore, de revêtir Rouen d'une image de modernité, réconciliant richesse du patrimoine passé et dynamisme présent. Si la construction et la diffusion de cette communication se fait à destination de l'extérieur, c'est-à-dire de la population touristique, elle est aussi largement assimilée par les habitants. À titre d'exemple, on citera la réaction locale, exprimée par le Club de la presse de Haute-Normandie qui s'est insurgé contre la prévision de destruction de Camille. Le Club a lancé, dès juillet 2010, une pétition pour s'y opposer et proposer de maintenir définitivement l'œuvre en place "parce que, explique-t-il, l'œuvre d'Arne Quinze est d'abord un formidable vecteur de communication pour les gens de médias que nous sommes ; [...] ; parce qu'à l'instar des Machines de Nantes, elle propulse Rouen, ville d'Art et d'Histoire, dans la modernité ; parce qu'elle piétonnise un pont entre deux rives qui continuent de s'ignorer poliment ; [...] parce que Camille est aussi peu consensuelle que le furent en leur temps les Nymphéas de 
Claude Monet et la Tour de Gustave Eiffel ; elle mérite de durer plus de deux mois ${ }^{(14) ”}$.

Ainsi, on lui octroie des fonctions en termes de pratiques (relier les deux rives), mais surtout de communication : elle a réussi à incarner l'image de la ville ("formidable vecteur de communication"; "elle propulse Rouen, ville d'Art et d'Histoire, dans la modernité") auprès des touristes et donc des populations résidentes, car on s'identifie pour beaucoup au travers de la façon dont on est vu. Prendre l'exemple des Machines, à Nantes, et plus encore de la tour Eiffel, à Paris, traduit véritablement le sentiment que l'œuvre est une composante identifiante majeure pour la ville. Même si elle n'a finalement pas été conservée (les œuvres d'Arne Quinze étant conçues pour être éphémères), cette émulation rappelle aussi l'importance des artistes, en tant qu'acteurs et vecteurs, dans la construction de l'image d'un lieu à destination des visiteurs comme des résidents permanents, les deux phénomènes étant intrinsèquement liés.

La mise en tourisme de Rouen suppose donc en amont un intérêt initial des touristes, qui choisissent toujours leur destination (Équipe Mit, 2005), mais aussi un encadrement fort des décideurs locaux, qui, en aval, orchestrent véritablement son essor. Aménagement, politiques des transports, construction et diffusion d'images sont des outils fondamentaux permettant une intégration du tourisme dans la ville historique, qui la redéfinit. En effet, ce dernier engendre des transformations réappropriées par ses habitants permanents, qui aspirent à jouir des "beaux lieux" de Rouen, au même titre que les visiteurs. Cependant, ces politiques de valorisation nécessitent de lourds investissements et des choix qui ne sont pas sans conséquences sur l'organisation à venir de la ville, et posent la question du caractère "soutenable" sur le long terme du processus.

\section{Une "touristification" soutenable sur le long terme?}

La mise en tourisme de la ville historique de Rouen se traduit par des politiques conciliant et réconciliant l'intérêt des résidents et des touristes. Cependant, au-delà de ce constat général, il convient d'étudier plus en détail quelles populations sont véritablement avantagées, derrière ces termes génériques. Si l'on prend l'exemple représentatif de Rouen sur mer, qui a réussi à drainer presque autant de visiteurs que de Rouennais, on observe que la répartition entre les quartiers de la ville n'est pas homogène. Si les habitants des rives gauche $(28 \%)$ et droite (22\%) se retrouvent effectivement sur la plage, les populations les moins favorisées des Hauts de Rouen y viennent encore peu (5\%). Ainsi, l'objectif initial de la collectivité locale d'offrir aux foyers modestes, ne pouvant partir à la mer, une compensation au sein de la ville n'est pas totalement atteint puisque Rouen sur mer demeure principalement fréquenté par les résidents du centreville et les touristes. Certes, cet évé- nement n'est pas totalement ignoré des habitants des banlieues résidentielles des Hauts de Rouen, dont la jeunesse participe activement à certaines animations, telles que la plongée. Néanmoins, il n'a globalement pas réussi à rassembler l'ensemble des populations rouennaises autour de l'agrément de la plage, pourtant populaire et gratuite.

Par ailleurs, toujours dans le cadre des questionnements quant aux bénéficiaires des actions permettant la mise en tourisme de Rouen, on peut s'interroger sur l'évolution en cours des quartiers de la rive gauche. Ceux-ci se caractérisent aujourd'hui par une mixité sociale, associant familles modestes et plus aisées. Or, la volonté de la municipalité de les réintégrer dans l'hypercentre historique et touristique ne risque-t-elle pas de transformer la structuration de leur population ? Certes, cette évolution est réclamée par les habitants de la rive gauche, qui se sentent délaissés par rapport à ceux de l'autre berge ${ }^{(15)}$. Néanmoins, ont-ils véritablement conscience qu'ils pourraient ne pas tous profiter de la réintégration de leurs quartiers dans le centre-ville, qui risquerait de provoquer leur gentrification, un phénomène très souvent associé à la touristification et à la patrimonialisation des sites (Knafou, 2007, p. 18) ? Et qui ne serait, d'ailleurs, qu'amplifié par le projet d'installer une importante gare TGV sur cette même rive, toujours dans le but de la revitaliser...

On observe donc que le processus d'intégration de la rive gauche, par 
son absorption dans l'hypercentre touristique et historique, pose la question de la population qui en bénéficiera. Certes, l'image de ces quartiers en sera améliorée et ils deviendront plus attractifs. Cependant, de cette situation découlera très probablement l'inflation de l'immobilier, qui repoussera les foyers modestes en périphérie, tandis que le centre-ville concentrera plus encore les populations privilégiées, comme sur la rive droite.

Enfin, on ne peut que constater le très fort engagement de la collectivité locale dans la mise en tourisme de Rouen. Cependant, celui-ci induit de lourds investissements, même si l'apport des partenaires financiers est important en fonction des opérations. L'entretien du patrimoine, la revalorisation des quais, le réaménagement de la rive gauche, les travaux du tramway et du TEOR se chiffrent en dizaines de millions d'euros, supportés par la ville et l'agglomération. De même, la politique d'événements de grande envergure, organisés tout au long de l'année, engendre des coûts très élevés pour la collectivité locale, dépassant le million d'euros, et ce, sans compter les frais internes de fonctionnement ${ }^{(16)}$. Certes, les retombées économiques locales sont en conséquence, comme on a pu l'observer, avec une forte augmentation de la fréquentation des sites touristiques de la ville, mais aussi des hôtels et des commerces. L'inscription du tourisme dans la ville historique de Rouen semble donc porter ses fruits. Toutefois, on peut s'interroger sur la viabilité à long terme de la poursuite de ce processus associant patrimoine, arts contemporains, événementiels, aménagements, modernisation des transports, tout en misant sur l'accessibilité et la gratuité, en raison des très importants coûts qu'il engendre...

\section{CONCLUSION}

L'étude de Rouen permet de comprendre davantage les processus de mise en tourisme d'une ville historique, évoluant sur deux siècles. Elle résulte de la combinaison de plusieurs facteurs qui placent les touristes au cœur du double phénomène de touristification et de patrimonialisation, depuis le XIX siècle. Ils assument le rôle de sélection des lieux, mais aussi de leur codification et de leur diffusion, par leurs récits écrits et oraux, voire leurs œuvres littéraires et picturales. C'est la ville définie au travers de ses lieux du patrimoine, identifiés et codifiés, que les générations futures de visiteurs viennent contempler et que les habitants permanents se réapproprient.

Le rôle des touristes est donc fondamental dans la mise en tourisme d'une ville historique, mais il n'est pas suffisant pour assurer la pérennisation de cette dynamique, dans un monde touristique désormais très concurrentiel. La forte implication des décideurs locaux s'avère aussi nécessaire, ainsi que l'adhésion au processus des populations résidentes. Ce phénomène, qui caractérise tous les lieux touristiques, est d'autant plus vrai dans une ville historique, car la richesse du patrimoine n'est pas toujours un atout. En effet, les réglementations de conservation que cette richesse induit, mais aussi l'image passéiste dont elle est souvent vecteur, peuvent nuire à l'inscription de la ville dans le présent et l'avenir. Afin d'éviter ce phénomène de muséification, la collectivité locale de Rouen a misé sur l'événementiel, qui, par son caractère éphémère, invite à une réinvention permanente de la ville, mais aussi sur l'art contemporain, pour perpétuer sa dimension culturelle sans la limiter aux grands artistes des siècles révolus (romantiques, impressionnistes).

Si l'association de ces deux composantes - événementiel et art contemporain - a pour dessein de façonner l'image d'une ville historique non sanctuarisée auprès des populations touristiques et permanentes, l'intégration du tourisme en son sein émane de politiques plus générales. En effet, touristification et dynamisation de la ville historique sont si intrinsèquement liées qu'elles dépendent d'un aménagement global, incorporant la protection et l'entretien du patrimoine, l'embellissement et la requalification des quartiers, mais aussi l'accessibilité. Ce dernier point relève d'une réflexion sur les moyens de communication et les transports publics. Rouen s'est démarquée pour avoir très tôt rendu piétonnier son centreville historique, mais aussi pour s'être dotée d'un tramway en partie sous-terrain. Au XXI siècle, l'agglomération reste dans l'air du temps, s'étant enrichie d'un réseau de bus 
très performant (TEOR), tandis que la municipalité soutient les déplacements à vélo, grâce à la mise en place d'un libre service de location (Cy'clic) disposant de stations disséminées dans la ville.

Ainsi, l'inscription du tourisme dans la ville historique est si profonde qu'elle fait partie de ses composantes inhérentes et influence ses modes d'“habiter" (Lévy, 2008), aussi bien pour les touristes que pour les résidents permanents. Les lieux polyvalents se multiplient, alors que les Rouennais se réapproprient les sites et animations, emblèmes de la ville aux yeux des visiteurs. Ce phénomène atteint une telle importance qu'il n'est plus possible de dissocier les actions menées pour l'une et l'autre de ces deux populations, touristique ou résidente.

Toutefois, ces choix politiques d'orchestration de la mise en tourisme de la ville historique posent la question de sa viabilité sur le long terme. En effet, ils induisent des investissements économiques très lourds, ainsi que des projets de développement urbain conséquents. Si les retombées locales sont avérées à Rouen, on peut s'interroger sur le caractère "soutenable" de ces importants budgets à longue échéance pour la collectivité, mais aussi sur la répartition de ces bénéfices. Apportent-ils une réelle plusvalue, en termes économiques et sociaux, à l'ensemble des habitants ? ou vont-ils contribuer à renforcer les discriminations, notamment par la gentrification d'anciens quartiers mixtes, voire populaires?
Ces enjeux économiques et sociaux, exprimés dans le cadre d'une politique aspirant à concilier préservation des richesses passées et dynamisme futur, concernent, audelà de Rouen, nombre de villes historiques et touristiques. Cette analyse appelle donc à la comparaison avec d'autres situations en France, en Occident, mais aussi dans les pays émergents du tourisme (Chine, Indonésie, Inde...), très sensibilisés aux problématiques d'association du patrimoine au développement économique et social. L'étude comparative de la mise en tourisme de leurs villes historiques, dans des temporalités qui leur sont propres, compléterait donc cette analyse, en permettant l'amorce d'une modélisation des processus.

NOTES

( I) Comme en témoignent les écrits des romantiques se prêtant aux voyages touristiques reposant sur la visite d'une succession de villes étapes. À titre d'exemple, Itinéraire de Paris à Jérusalem, de Chateaubriand ( 852 [I RE édition |8/ I]) - qui, selon ses propres termes en 1827, "senvit de guide à une foule de voyageurs" - décrit son périple passant par les cités historiques célèbres de Méditerranée (Athènes, Tunis, Le Caire,

Constantinople, Jérusalem...).

(2) Eté 2010 : un été impressionnant, dossier de presse municipal de la ville de Rouen, septembre 2010.

(3) Rouen sur mer 2010, point au 26 juillet 2010, enquête élaborée et menée par la municipalité de Rouen.

(4) Phénomène qui n'est pas nouveau, comme en attestent les guides touristiques des stations depuis la fin du XIX siècle

(Pickel, 2004).

(5) [http://wmw.annuaire-

mairie.fr/monument-historique-rouen.html].

(6) Entretien avec Lionel Guéret-Laferté,

directeur des manifestations publiques de la ville de Rouen (juillet 2010).

(7) Estimation de la fréquentation de l'Armada en 2008, grâce à des comptages effectués à l'aide de caméras par la municipalité.

(8) Le réseau des chambres de commerce et d'industrie de Normandie (2009), Un mois après: le bilan de l'édition de 2008 de l'Armada [http://www.drakkaronline.com]. (9) Rouen sur mer, point du 26 juillet 2010, enquête menée par la municipalité de Rouen.

(10) Été 2010 : un été impressionnant, dossier de presse municipal de la ville de Rouen, septembre 2010.

(I I) Rémy Knafou rappelle ce phénomène, en soulignant que la ville est "à la fois lieu d'impulsion du goût des voyages, mais aussi point de départ, lieu de passage, lieu d'arrivée ou de séjour" (2007, p. 9.)

(I2) [http://www.rouen.fr/cyclic].

(13) Rouen impressionnée 3 juillet-26 août 2010 [http://rouenimpressionnee.fr/presentation].

(14) Club de la presse de HauteNormandie, 9 juillet 2010, "II faut sauver Camille!", Association des professionnels des médias et de la communication [http://www.clubpresse-hn.com].

(I5) Entretien avec Lionel Guéret-Laferté, directeur des manifestations publiques de la ville de Rouen (28 octobre 2010).

(16) Ce ne sont pas les budgets consolidés, que la municipalité a encore du mal à évaluer en raison de la multiplicité des frais occasionnés. 


\section{RÉFÉRENCES BIBLIOGRAPHIQUES}

Jean-Pierre BABELON et André CHASTEL, La Notion de patrimoine, Liana Levi, 2000.

Catherine BeRTHO-LAVENIR, La Roue et le Stylo : comment nous sommes devenus touristes, Odile Jacob, 1999.

Dominique Bouluier, La Ville événement. Foules et publics urbains, "La ville en débat", Puf, 2010.

François René DE ChATEAUBRIAND, Itinéraire de Paris à Jérusalem, Librairie Firmin Didot frères, 1852 (Ire édition |8| I). Été 2010 : Un été impressionnant, Dossier de presse municipal de la ville de Rouen, septembre 2010.

Philippe Duhamel et Rémy Knafou, Mondes urbains du tourisme,

Belin, 2007

Équipe Mit, Tourisme I. Lieux communs, Belin, 2002.

Équipe Mit, Tourisme 2. Moments de lieux, Belin, 2005.

Rémy KNAFOU, "L'urbain et le tourisme, une construction

laborieuse", dans Philippe DUHAmel et Rémy KNAFOu, Mondes urbains

du tourisme, Belin, 2007.

Christine Laroche et Philippe Hermet, "De la prise en compte du tourisme dans le succès d'un projet de ville", dans Thomas LAMAND (dir.), Ville, urbanisme et tourisme, "Cahiers Espaces", n I04, ETE, 2010.

Olivier LazZarottı et Sylvine PICKEL, "Lieux remplis, lieux vidés : temporalités touristiques", dans Jacques LÉvy (dir.), Les Échelles de l'habiter, PUCA, 2008.

Olivier LAZZAROTTI et Philippe VIOLIER, "Par-delà le patrimoine et le tourisme", dans Olivier LAZZAROTTI et Philippe VIOLIER, Tourisme et patrimoine. Un moment du monde, Presses de l'université d'Angers,

2007.

Jacques Lévy (dir.), Les Échelles de l'habiter, PUCA, 2008.

Jacques LÉVY, "Ville", dans Jacques LévY et Michel LusSAUlt (dir.),

Dictionnaire de la géographie et de l'espace des sociétés, Belin, 2003.

Observatoire régional du tourisme Normandie, Guide statistique du tourisme normand, 2006 [en ligne]. À partir de [http ://www.normandy-tourism.org/w/pdf/guid_stat/guid_stat_2006.pdf].

Sylvine PICKEL, Représentations et pratiques de la nature dans les stations atlantiques françaises. Une construction sociale évolutive, thèse de doctorat de géographie, Université Paris VII- Denis Diderot, 2004.
Laurent SALOMÉ, Une ville pour l'impressionnisme. Monet, Pissarro et Gauguin à Rouen, Skira Flammarion, Musée des Beaux-Arts de Rouen, 2010.

Marie-Anne SIRE, La France du patrimoine : les choix de la mémoire, Gallimard, 2005.

Mathis Sтоск, "Habiter touristiquement la ville", dans Philippe DuHAmel et Rémy Knafou, Mondes urbains du tourisme, Belin, 2007. Philippe Violier et Antonio Manuel Zarate, "Politiques urbaines du tourisme", dans Philippe DuHAMEL et Rémy KNAFOU, Mondes urbains du tourisme, Belin, 2007.

Vincent VLÈs, Politiques publiques d'aménagement touristique. Objectifs, méthodes, effets, "Le territoire et ses acteurs", Presses universitaires de Bordeaux, 2006. 